Roland Heuermann

Strategisches IT-Management 



\section{Roland Heuermann}

Strategisches

IT-Management

In Privatwirtschaft und Verwaltung

OLDENBOURG 
Lektorat: Dr. Stefan Giesen

Herstellung: Tina Bonertz

Grafik: thinkstockphotos.com

\section{Bibliografische Information der Deutschen Nationalbibliothek}

Die Deutsche Nationalbibliothek verzeichnet diese Publikation in der Deutschen Nationalbibliografie; detaillierte bibliografische Daten sind im Internet über http://dnb.dnb.de abrufbar.

\section{Library of Congress Cataloging-in-Publication Data}

A CIP catalog record for this book has been applied for at the Library of Congress.

Dieses Werk ist urheberrechtlich geschützt. Die dadurch begründeten Rechte, insbesondere die der Übersetzung, des Nachdrucks, des Vortrags, der Entnahme von Abbildungen und Tabellen, der Funksendung, der Mikroverfilmung oder der Vervielfältigung auf anderen Wegen und der Speicherung in Datenverarbeitungsanlagen, bleiben, auch bei nur auszugsweiser Verwertung, vorbehalten. Eine Vervielfältigung dieses Werkes oder von Teilen dieses Werkes ist auch im Einzelfall nur in den Grenzen der gesetzlichen Bestimmungen des Urheberrechtsgesetzes in der jeweils geltenden Fassung zulässig. Sie ist grundsätzlich vergütungspflichtig. Zuwiderhandlungen unterliegen den Strafbestimmungen des Urheberrechts.

(C) 2014 Oldenbourg Wissenschaftsverlag GmbH Rosenheimer Straße 143, 81671 München, Deutschland

www.degruyter.com/oldenbourg

Ein Unternehmen von De Gruyter

Druck und Bindung: $\mathrm{CPI}$ books $\mathrm{GmbH}$, Leck

Gedruckt in Deutschland

Dieses Papier ist alterungsbeständig nach DIN/ISO 9706.

ISBN 978-3-486-71807-2

e-ISBN 978-3-486-85548-7 